Original Article

\title{
Effects of visual fatigue caused by smartphones on balance function in healthy adults
}

\author{
Young-Hyun Park, PT, MSc ${ }^{1,3)}$, Chang-Man An, PT, MSc ${ }^{1,3)^{*}}$, Sung-Jun Moon, PT, MSc ${ }^{2,3)}$ \\ 1) Department of Physical Therapy, College of Medical Sciences, Hanseo University: Hanseo 1-ro, \\ Haemi-Myun, Seosan-Si, Chungcheongnam-do 31962, Republic of Korea \\ 2) Department of Rehabilitation Science, Graduate School, Daegu University, Republic of Korea \\ 3) Department of Physical Therapy, Chonbuk National University Hospital, Republic of Korea
}

\begin{abstract}
Purpose] The purpose of this study was to determine the effects of visual fatigue caused by smartphone use on balance function. [Subjects and Methods] The participants consisted of 22 healthy male and female adults. Their postural stability, limit of stability, and limit of stability running time were evaluated using a computerized posturography apparatus before and after inducing visual fatigue. Postural stability and the limit of stability were divided into static and dynamic conditions. [Results] There were significant differences between the dynamic postural stability, the static and dynamic limit of stability, and both the static and dynamic limit of stability running times after the induction of visual fatigue. [Conclusion] The results showed that visual fatigue caused by smartphone use has a negative effect on balance function. Therefore, reducing visual fatigue through proper rest is necessary.

Key words: Smartphone, Visual fatigue, Balance function
\end{abstract}

(This article was submitted Oct. 6, 2016, and was accepted Oct. 27, 2016)

\section{INTRODUCTION}

Other than its primary purpose of telecommunication, smartphones have advantages as portable devices allowing connection to the internet, games, and online communities ${ }^{1}$. However, because smartphone technology is based on blue light, there is a high probability of damaging vision due to the shorter wavelength of light when used for a long period of time ${ }^{2}$. From the research on ocular fatigue induced by multimedia aimed toward the general public, it has been discovered that liquid crystal displays induce dry eye symptoms more frequently than ordinary books and the displays increase the level of visual fatigue from feeling dazzled ${ }^{3}$. If the visual fatigue continues in everyday life, it can affect visual processing and can cause various problems such as ocular pain, damaged corneal epithelial cells, conjunctival hyperemia, and decreased visual acuity. This continued visual fatigue also has a negative effect on visual feedback processing that integrates the vestibular organ system with the somatosensory network and can further cause an interruption in the body's postural control system ${ }^{4-6)}$. Vision as part of the postural control system may vary depending on ground stability and visual task. Therefore, the purpose of this study was to determine the effects of visual fatigue from smartphones on balance function depending on ground stability and visual task level and to provide smartphone users basic vision care information for preventive measures.

\section{SUBJECTS AND METHODS}

This study included 22 young healthy adults (11 males and 11 females with an average height of $169.9 \pm 7.8 \mathrm{~cm}$ and an average weight of $62.7 \pm 13.8 \mathrm{~kg}$ ) after they provided informed consent to participate in this study. The study was conducted

\footnotetext{
*Corresponding author. Chang-Man An (E-mail: dks3597@hanmail.net)

C2017 The Society of Physical Therapy Science. Published by IPEC Inc.

This is an open-access article distributed under the terms of the Creative Commons Attribution Non-Commercial No Derivatives (by-nc-nd) License $<$ http://creativecommons.org/licenses/by-nc-nd/4.0/>.
} 
Table 1. General patient characteristics $(\mathrm{N}=22)$

\begin{tabular}{lcc}
\hline Characteristics & Mean $\pm \mathrm{SD}$ & Range \\
\hline Gender $(\mathrm{M} / \mathrm{F})$ & $11 / 11$ & \\
Age $($ years $)$ & $25.3 \pm 3.8$ & $22-29$ \\
Height $(\mathrm{cm})$ & $169.9 \pm 7.8$ & $185-153$ \\
Weight $(\mathrm{kg})$ & $62.7 \pm 13.8$ & $90-46$ \\
\hline
\end{tabular}

Values are expressed as mean $\pm \mathrm{SD}$

M: male; F: female
Table 2. Comparison of postural control before and after inducing visual fatigue $(\mathrm{N}=22)$

\begin{tabular}{lcc}
\hline Value & $\begin{array}{c}\text { Before visual } \\
\text { fatigue }\end{array}$ & $\begin{array}{c}\text { After visual } \\
\text { fatigue }\end{array}$ \\
\hline Static PS & $0.36 \pm 0.2$ & $0.38 \pm 0.2$ \\
Dynamic PS & $0.82 \pm 0.4$ & $1.02 \pm 0.6^{* *}$ \\
Static LOS (\%) & $60.32 \pm 13.9$ & $51.96 \pm 15.7^{* *}$ \\
Dynamic LOS (\%) & $35.68 \pm 11.5$ & $29.55 \pm 12.2^{* *}$ \\
Static LOS running time (sec) & $35.55 \pm 8.4$ & $40.5 \pm 14.2^{*}$ \\
Dynamic LOS running time (sec) & $47.36 \pm 9.5$ & $58.1 \pm 20.7^{*}$ \\
\hline
\end{tabular}

Values are expressed as mean $\pm \mathrm{SD}$

PS: postural stability; LOS: limit of stability

$* \mathrm{p}<0.05, * * \mathrm{p}<0.01$

in accordance with the ethical principles of the Declaration of Helsinki and the local research ethics committee approved the study protocol.

Individuals with a history of ophthalmological injury or disease that may affect tear secretion, who have had surgery or with a disorder that may affect postural control, have had LASIK surgery, were diagnosed with xerophthalmia, or who wear contact lenses were excluded. The size of the smartphones was limited to 4.7-5.7 inches and the equipment type was limited to either the GALAXY NOTE 5 (SM-N920S), the LG G5 (F-700S), or the iPhone 6S (A-1688).

After sufficient rest without visual fatigue was given, subjects were positioned upright with their arms on a table and the smartphones were consistently held $40 \mathrm{~cm}$ away from the eyes ${ }^{7)}$. Viewing time to induce the visual fatigue was limited to 40 minutes ${ }^{8}$.

Computerized posturography balance data were collected using the Biodex Balance System SD (BBS, Biodex Medical System, Inc., New York, USA). The BBS was used to measure the postural stability (PS) and the limit of stability (LOS). Subjects were positioned on the apparatus with their two hands together and their feet apart to adjust the center of pressure (COP) so that it was at the center of the concentric circle. Measurements were calculated from the process of the participants maintaining or moving their COP.

The subjects were instructed to maintain their COP at the center of the concentric circle which was indicated on the monitor in order to measure static and dynamic PS before and after the induction of visual fatigue. Three trials where two measurements were made during a $20 \mathrm{~s}$ period at $10 \mathrm{~s}$ intervals were completed by the subjects and the measurements were averaged over the three trials. The dynamic PS was measured while the participant controlled the unfixed footboard that provided 12 different levels of stability, levels 12 to 1, decreasing from 12, which was the most stable, to 1, the least stable. The apparatus was set at level 5 for the static PS experiment. The stability index scores were calculated from the degree of tilt deviation from horizontal; the lower postural stability index score indicated superior balance function.

For static LOS measurements before and after the induction of visual fatigue, the footboard was fixed and eight randomly positioned markings flashed around the reference point in the center of the monitor. Measurements were made as the participants changed the position of the COP in response to the eight signals; the return time was calculated. The dynamic LOS was measured while controlling the unfixed footboard at 12 different levels, level 12 to 1 , with decreasing stability. The apparatus was set at level 5 for assessment of static LOS, and measurements were calculated using the same method. The static and dynamic LOS running time were recorded. The higher LOS score and shorten running time indicated superior balance function.

The general characteristics of the participants were characterized as the average \pm standard deviation (SD). The Kolmogorov-Smirnov test was used to analyze the differences in PS, LOS, and LOS running time before and after the induction of visual fatigue. The paired t-test was used for comparisons of normally distributed data sets. The data were analyzed with PASW statistical software ver. 18.0 (SPSS Inc., Chicago, IL, USA) using $\mathrm{p}<0.05$ as the level of significance.

\section{RESULTS}

Patient characteristics are listed in Table 1. The overall stability index (OSI) assessing the changed COP from all directions was used, with the lowest score referring to good postural balance control and sufficient PS. The OSI of static PS before inducing visual fatigue $(0.36 \pm 0.17)$ was not significantly different than PS after inducing visual fatigue $(0.38 \pm 0.2)$. However, the OSI of dynamic PS significantly increased from before $(0.82 \pm 0.4)$ to after $(1.02 \pm 0.59)$ the induction of visual fatigue $(\mathrm{p}<0.01)$ (Table 2).

The directional control score (DCS) assessed the degree of COP control with a higher DCS indicating better performance during directional COP control. The DCS of static LOS significantly decreased from $60.32 \pm 13.88$ before visual fatigue induction to $51.96 \pm 15.68$ after induction $(\mathrm{p}<0.01)$. The DCS of dynamic LOS changed significantly from $35.68 \pm 11.51$ to $29.55 \pm 12.19$ before and after the visual fatigue induction, respectively $(\mathrm{p}<0.01)$ (Table 2$)$. 
The static LOS running time increased significantly after visual fatigue induction from $35.55 \pm 8.38 \mathrm{~s}$ before to $40.5 \pm$ $14.19 \mathrm{~s}$ after $(\mathrm{p}<0.05)$. The dynamic LOS running time also significantly increased from $47.36 \pm 9.5 \mathrm{~s}$ to $58.05 \pm 20.7 \mathrm{~s}$ before and after inducing visual fatigue, respectively $(\mathrm{p}<0.05)$ (Table 2$)$.

\section{DISCUSSION}

This study investigated the effects of visual fatigue from using smartphones on postural control in 22 healthy adults. Visual fatigue was induced by placing smartphones $40 \mathrm{~cm}$ from the eyes for $1 \mathrm{hr}$. Static PS, dynamic PS, static LOS, dynamic LOS, static LOS running time, and dynamic LOS running time before and after the induction of visual fatigue were measured using BBS. Postural control ability during dynamic PS $(p<0.01)$, static LOS $(p<0.01)$, and dynamic LOS $(p<0.01)$ after induction of visual fatigue significantly decreased but there were no significant changes in static PS. This can indicate that the static PS results in this study might have been affected by the measurement duration of $20 \mathrm{~s}$, which may not be long enough to effectively modulate visual fatigue. Shu et al. ${ }^{9}$ showed that the maximum time a person can hold their blinking eyes with no visual fatigue was $23.12 \mathrm{~s}$, but it was shortened to $17.09 \mathrm{~s}$ after $1 \mathrm{hr}$ of computer work, reducing the ocular protection index. For LOS running time in this study, both static LOS and dynamic LOS running time significantly increased $(p<0.05)$, confirming that postural control ability was diminished after the induction of visual fatigue. Therefore, visual fatigue may also substantially affect other studies such as the visual perception feedback study, where data is obtained regarding visual focusing ability, or in the mobility induced setting or the visual searching study which measured the level of attention ${ }^{10)}$. Won et al. ${ }^{11)}$ showed that changes in visual processing can affect the degree of dependence on proprioception and postural sway can be increased if visual processing is obstructed. The limitation of this study was the limited number of participants who had no balance deficits (22 young adults aged in the twenties). Also, It was unable to control the visual fatigue that may occur in the evaluation process BBS. Further studies should be carried with a wide age range of subject through an objective indicator of the visual fatigue index. In conclusion, the results of this study demonstrated that visual fatigue caused by smartphone use interferes with the visual ability to control posture and possibly reduces the ability to balance. Thus, this study suggests that it is necessary for smartphone users to reduce visual fatigue by taking appropriate rest. Also, proper guidelines on the use of smartphones should be proposed on various symptoms caused by visual fatigue from smartphones.

\section{REFERENCES}

1) Park YM: A study on adult's smartphone addiction and mental health [dissertation]. Sanji University, 2011 (in Korean).

2) Kang SY, Hong JE, Choi EJ, et al.: Blue-light induces the selective cell death of photoreceptors in mouse retina. J Korean Ophthalmic Opt Soc, 2016 , 21: 69-76. [CrossRef]

3) Jang YS, Kim US, Baek SH, et al.: Research on ocular fatigability induced by multimedia and instrument features. J Korean Ophthalmol Soc, 2012, 53: 689-693. [CrossRef]

4) Park HJ, Yi K: Relationship between middle school student's computer using time and dry eye. J Korean Ophthalmol Soc, 2002 , 43: 449-454.

5) Lateiner JE, Sainburg RL: Differential contributions of vision and proprioception to movement accuracy. Exp Brain Res, 2003, 151: 446-454. [Medline] [CrossRef]

6) Shumway-Cook A, Woollacott M: Motor control: translating research into clinical practice, 3rd ed. Philadelphia: Lippincott Williams \& Wilkins, 2007.

7) Lee SH: Evaluating the influence of smart phone use on the psycho-physiological condition [dissertation]. Seoul: Konkuk University, 2010 (in Korean).

8) Park JS, Choi MJ, Ma JE, et al.: Influence of cellular phone videos and games on dry eye syndrome in university students. J Korean Acad Community Health Nurs, 2014, 25: 12-23. [CrossRef]

9) Suh YW, Kim KH, Kang SY, et al.: The objective methods to evaluate ocular fatigue associated with computer work. J Korean Ophthalmol Soc, 2010, 51: 1327-1332. [CrossRef]

10) Shen J, Reingold EM, Pomplun M: Guidance of eye movements during conjunctive visual search: the distractor-ratio effect. Can J Exp Psychol, 2003, 57: 76-96. [Medline] [CrossRef]

11) Won MH, Kim MC, Kim SJ, et al.: The effect of visual information provision on the changes of electromyogram activity in trunk and lower leg muscles during dynamic balance control. Korean J Sports Med, 2014, 32: 44-54. [CrossRef] 